\title{
CAD-based intelligent robot system integrated with 3D scanning for shoe roughing and cementing
}

\author{
Cheng-Chang Chiu ${ }^{1, *}$, Wen-Teng Wang ${ }^{2}$, and Wan-Shan Yin ${ }^{1}$ \\ ${ }^{1}$ Mechatronics Section, Metal Industries Research Development Centre, \\ ${ }^{2}$ Taiwan National Formosa University, Taiwan
}

\begin{abstract}
Roughing and cementing are very essential to the process of bonding shoe uppers and the corresponding soles; however, for shoes with complicated design, such as sport shoes, roughing and cementing greatly relied on manual operation. Recently, shoe industry is progressing to $3 \mathrm{D}$ design, thus $3 \mathrm{D}$ model of the shoe upper and sole will be created before launching into mass production. Taking advantage of the 3D model, this study developed a plug-in program on Rhino 3D CAD platform, which realized the complicated roughing and cementing route planning to be performed by the plug-in program, integrated with real-time 3D scanning information to compensate the planned route, and then converted to working trajectory of robot arm to implement roughing and cementing. The proposed 3D CAD-based intelligent robot arm system integrated with 3D scanning for shoe roughing and cementing is realized and proved to be feasible.
\end{abstract}

\section{Introduction}

Roughing and cementing are very essential to the process of bonding shoe uppers and the corresponding soles, especially for the cold cement construction of shoe manufacturing. The purpose of roughing before cementing is to help breaking the smooth surface for better adhesion and thus reaching the desired bonding strength. The upper and the sole are usually designed to be in complicated freeform surface, which causes shoe manufacturers in the past required and relied greatly on manual roughing and cementing. In the recent years, because of the severe increase of personnel cost, awareness of labor right, and frequent happened strikes in Southeast Asia, those Taiwan shoe manufacturers who allocated factories in oversea started striving for transforming to automation manufacturing, and thus the automatic roughing and cementing process would be the first step toward automation. Cold cement shoes can be divided into two types: cup sole which has tall side wall to cradle the upper of the shoes and flat sole without side wall, as shown in Fig. 1. These two types have very different sole design, and the work trajectories on shoe sole for roughing and cementing are differed; thus the automation for these two different sole types need to be separated designed. Flat sole is attached directly to the bottom of the slip-lasted shoe upper, hence the area for roughing and cementing will be the whole shoe bottom; as for cup sole, the area will be extended to the margin of an upper. The side roughing will be presented on the die line of the sole as the yellow curve line shown in Fig. 1(b).

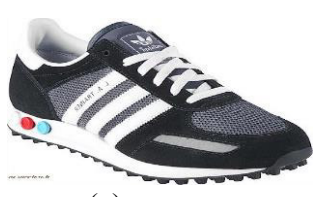

(a)

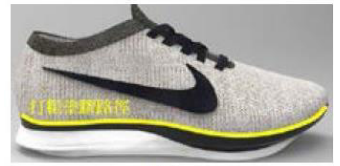

(b)
Fig. 1. Two sole types: (a) flat sole [1], and (b) cup sole [2].

Chuanyu $\mathrm{Wu}$ et al. developed a 5-axis apparatus where a 3D scanner and a work tool for roughing were fixed on the main axis respectively as well as a upper was positioned on the machine bed [3]. Yuzhen Jin et al. focused on the characteristics of shoe upper scanning and designed an infrared lasers 3D scanner specifically for shoe upper 3D measurement [4]. Chuanyu Wu integrated the achievements of [3] and [4] and further proposed a method to generate the work trajectory based on the obtained scanned shoe sole information. Francesco Jatta et al. designed a 6-DOF manipulator integrated with $\mathrm{CAD} / \mathrm{CAM}$ to realize automatic bottom and side roughing and cementing of shoe uppers [6]. The above mentioned approaches that generated roughing working trajectory from $\mathrm{CAD}$ model of the upper and did not take the error caused by the previous process into consideration, thus only applicable to the situation of small error. Zhongxu $\mathrm{Hu}$ et al. combined a 3D scanner to a mechanical arm and roughing the surface via high pressure grit blasting [7], which was consisted of a rotary table to position the upper, a 3D scanner fixed on the linear slide mechanism, and and automatic manipulated blasting nozzle.

Wen-Teng Wang et al. propose an algorithm to generate and control the trajectory of roughing and cementing for an intelligent roughing and glue spraying robot arm with 3D visualization. The proposed 
algorithm was adoptable to different shoe uppers by taking advantage of the 3D visualization to obtain the data of the differences of different shoe shapes, and calculated the couture area of the shoe bottoms and working trajectories to drive the robot arm to complete the process automatically. Due to the features of every shoe upper is considered, the automatic roughing and cementing processes can fulfill different characteristics and quality requirements of different shoe types.

Different from the 2D modeling of the traditional footwear industry, owing to the current development of 3D CAD system, the application of 3D modeling in shoe manufacturing has been widely adopted, such as some international big brands like Nike, Adidas, UA and etc. Therefore, today's shoe factory has already had 3D shoe model before launching into production, which enables the automatic generation of roughing and cementing working trajectory to cup soles with high side walls. Based on the existing 3D models, this paper took advantage of the open characteristics of Rhino 3D CAD platform to develop a plug-in program of $3 \mathrm{D}$ graphic interface for generating the working trajectory of roughing and cementing. Further combined with the $3 \mathrm{D}$ visualization scanner to retrieve the real information of each work piece to compensate and turn the route, and then drive the robot arm to execute the processes of roughing and cementing.

\section{Experiment and methodology}

In order to equip the auto roughing and cementing system with broaden applications and more friendly operations, this paper introduced the Rhino 3D CAD platform and developed a plug-in program to plan and generate the working trajectory after the information of $3 \mathrm{D}$ shoe models is retrieved. Meanwhile, combined with the real-time $3 \mathrm{D}$ visualization scanner and algorithm of route conversion, the robot arm is auto-driven to complete the processes of roughing and cementing. The experiment set-up of the proposed design is presented in Fig. 2. The end effector of the robot arm is installed with clamp to fetch a lasted upper. After the lasted upper is clamped, the lasted upper is fixed in vertical or certain inclination angle to IR laser of the 3D scanner, moved horizontally at a stable speed, and 3D scanner is activated to retrieve the information of the upper. Followed with the calculation of roughing and cementing trajectory, the format of the robot arm working path is converted and loaded to the controller of the robot arm to drive the robot arm and the lasted upper to compete the roughing and cementing with planned route.

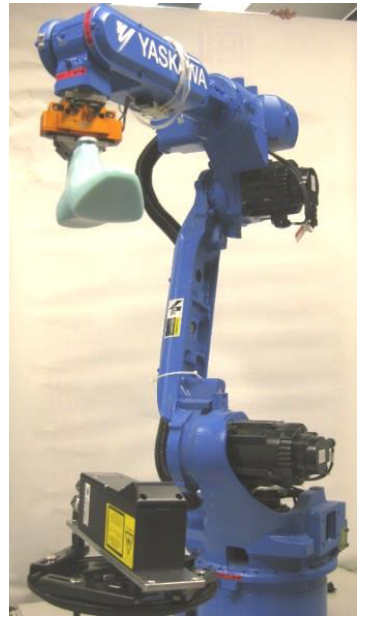

Fig. 2. Experiment set-up of the proposed design [8].

Figure 3 demonstrates the flow-chat of the proposed design, and the processes are elaborated as follows.

1. Activate Rhino 3D and load the plug-in roughing and cementing route planning program.

2. Start up the plug-in program and retrieve the 3D model of IGES format.

3. Plan the working trajectory with the curve rendering function of Rhino 3D.

4. Set the working trajectory and posture with plug-in program.

5. Output the working trajectory and posture.

6. Activate the modification program.

7. Read the working trajectory and posture.

8. Read the information of 3D visual scanner.

9. Perform the calculation of route compensation.

10. Output the route of robot arm.

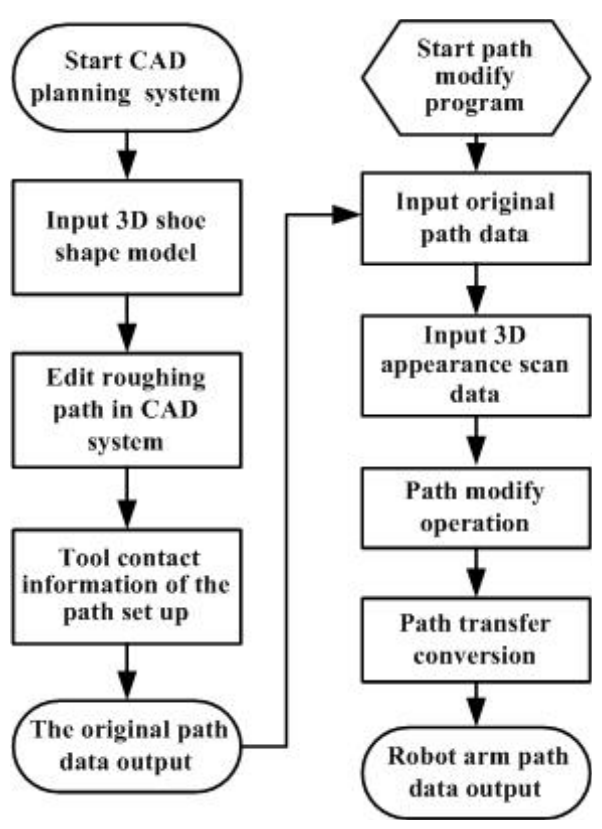

Fig. 3. Flow-chat of the proposed design. 


\section{Trajectory planning by 3D graphical interface of CAD platform}

Due to the high side wall, the roughing and cementing trajectory of a cup sole is not available directly from the shape of upper. In order to enable the auto roughing and cementing of cup soles, the 3D design model is adopted and imported to the proposed plug-in program to do the working trajectory planning. Rhino 3D possesses strong function in free curve model and surface rendering and is in very reason price, which has made it popular to shoe manufacturers. Besides, Rhino.Net SDK helps developers to extend Rhino's functionality using Rhino plug-ins. Rhino.Net SDK supports VB.NET, C\#, C++. NET, Delphi.NET and other programming languages to develop plug-ins. Due to Rhino.Net SDK itself uses $\mathrm{C}++$ to package the category library, $\mathrm{C}++$.Net would be the most direct and compatible option for plug-in development; thus, this study uses $\mathrm{VC}++2010$ for Rhino.Net SDK plug-in development.

\subsection{Operation and development of plug-in programs}

1. Load the plug-ins to Rhino 3D.

2. Read the IGES file for the shoe 3D model.

3. Use the Rhino 3D curve rendering feature.

4. Generate the contact points of the roughing and cementing trajectory.

5. Set curve segmentations of the roughing and cementing posture

6. Graphically set vectors of the sub-section for the roughing and cementing posture

7. Input the values of vectors of the sub-section for the roughing and cementing posture.

8. Output the trajectory of the contact points and posture.

\subsection{Background design of roughing and cementing plug-in}

In order to bring the reality of live operation and direct interaction with the hardware to users, this study designed to import the 3D model that is build according to the real size and position of the hardware to be the background of the plug-in, as shown in Fig. 4.

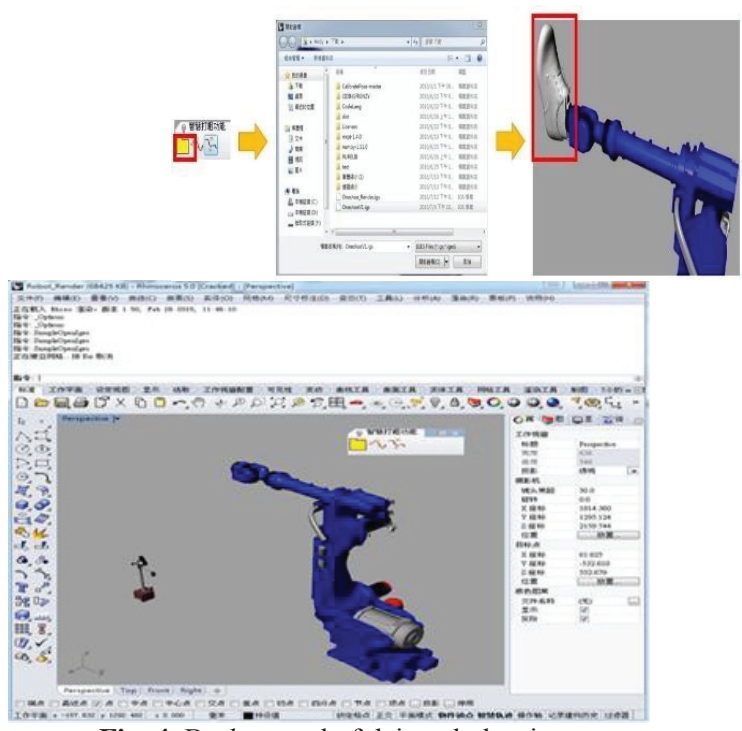

Fig. 4. Background of deigned plug-in.

\subsection{Functionality development of plug-in}

The functions of the plug-in program are developed by using $\mathrm{VC}++2010$ to refer to the API functions provided by Rhino.Net SDK, and the function algorithm is introduced into the plug-in program. Table 1 shows the main API functions referenced from Rhino.Net SDK in this study.

Table 1. Main API functions used by plug-in program

\begin{tabular}{|c|c|}
\hline Function & Corresponding Functions \\
\hline $\begin{array}{l}\text { Read } \\
\text { IGES files }\end{array}$ & $\begin{array}{l}\text { 1. } / / \text { define model's format in IGES } \\
\text { const wchar_t* szFilter = L"IGES Files (*.igs;*.iges) }\left.\right|^{*} . \text { igs; } \\
\text { *.iges||"; } \\
\text { 2. } \\
\text { //show dialog to choose a model } \\
\text { CFileDialog dialog( TRUE, L"igs", 0, dwFlags, szFilter, } \\
\text { pParentWnd, 0, TRUE ); } \\
\text { 3. } / / \text { script an imported model } \\
\text { script.Format( L"_-Import } \backslash " \% s \backslash " \text { Enter_Enter_Enter", } \\
\text { filename ); }\end{array}$ \\
\hline $\begin{array}{l}\text { Generate } \\
\text { contact } \\
\text { points for } \\
\text { roughing } \\
\text { and } \\
\text { cementing }\end{array}$ & $\begin{array}{l}\text { 1. //choose a desired curve } \\
\text { CRhinoGetObject go; } \\
\text { go.SetCommandPrompt( L"Select curve" ); } \\
\text { //get the curve's length } \\
\text { CRhinoGetNumber gn; } \\
\text { gn.SetCommandPrompt( L"Length from start" ); } \\
\text { gn.SetLowerLimit( 0.0, TRUE ); } \\
\text { gn.SetUpperLimit( crv_length, TRUE ); } \\
\text { gn.GetNumber(); } \\
\text { if( gn.CommandResult() != success ) } \\
\text { return gn.CommandResult(); } \\
\text { double length = fabs( gn.Number( ) ); } \\
\text { //get the curve's length } \\
\text { ON_3dPoint pt = crv->PointAt( length ); } \\
\text { context.m_doc.AddPointObject( pt ); }\end{array}$ \\
\hline $\begin{array}{l}\text { Rhino } \\
\text { Command } \\
\text { prompt }\end{array}$ & $\begin{array}{l}\text { gn.SetCommandPrompt( L"Do you want to modify the } \\
\text { vector?(Yes:0/No:1)" ); }\end{array}$ \\
\hline
\end{tabular}

\subsection{Function of designed plug-in}

The process of the roughing and cementing trajectory planning by plug-in program is explained as follows. 
1. Read the IGES format file of the 3D model. IGES is the standard exchange format of geometric information for CAD/CAM system, thus $3 \mathrm{D}$ model is format in IGES in this paper. Figure 5 shows the operation procedure and results of the plug-in.
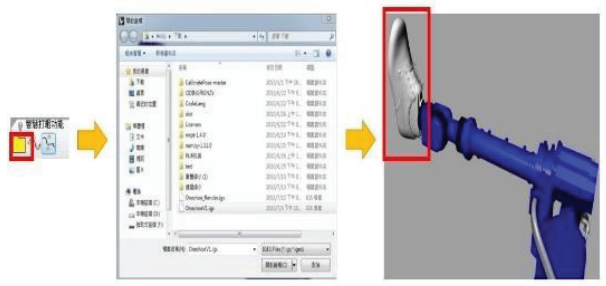

Fig. 5. Plug-in program reads 3D shoe model.

2. Using Rhino 3D graphic interface to render the contours of roughing and cementing area in the existed 3D model. For flat-bottom shoe type, the planned contour would be the max peripheral profile of the bottom. For the cup sole shoe type, the planned contour would be the die line of the 3D shoe sole model which mapped on the upper. Figure 6 is the planned contour for the cup sole type shoe.

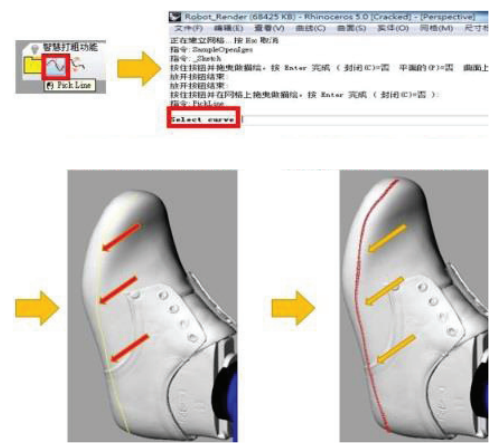

Fig. 6. Contour planning for cup sole shoe type.

3. Vector setting for the segments of the roughing and cementing contour posture. Plug-in program is employed to select the segmentation points on the contour, as shown in Fig. 7.
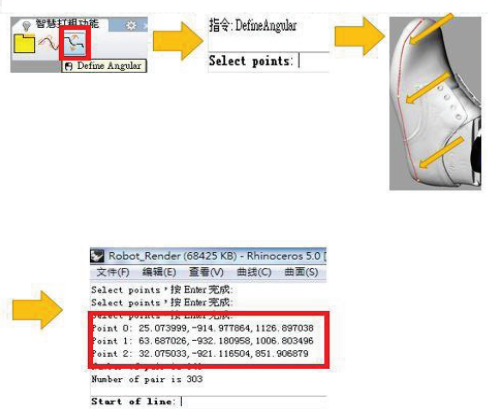

Fig. 7. Segmentation point selection.

4. Set the vectors of the contact posture of each segment. The contact angle of roughing on the segments of the upper may be different; the plug-in can be further developed to individually set the information for the specific segment. Figure 8 shows the vector setting of the segmented contours.

5. Output the contact points and the corresponding vectors for the roughing and cementing posture.

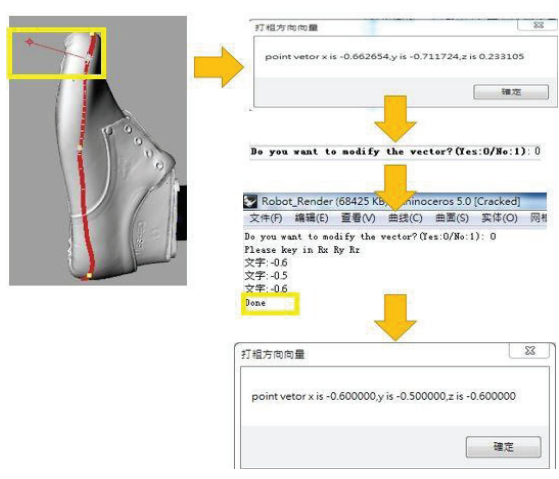

Fig. 8. Vectors setting of each segment of contour.

\section{3D Visualization}

In practice, there existed errors in the previous process of upper manufacturing; therefore, the trajectory planning requires modification and compensation with the 3D geometry information of the upper retrieved by the $3 \mathrm{D}$ scanner. When the scanning is completed, the $3 \mathrm{D}$ coordinator data originated at the $3 \mathrm{D}$ scanner is obtained and then real-time transformed to origin of the coordinator to the end effector of the robot arm.

\subsection{Application of 3D visualization}

The proposed system is developed to fulfill the requirement of small quantity, customization, and fast production of footwear manufacturing; hence, the system platform is designed to be equipped with the $3 \mathrm{D}$ visualization system Gocator 2340 to scan the lasted upper clamped by the robot arm. The $3 \mathrm{D}$ scanner is fixed on the platform; the robot arm clamps the lasted upper and move toward the scanner and then move horizontally in stable velocity for scanning the lasted upper, shown as Fig. 3.

\subsection{D scanning of cup sole shoe type}

In order to retrieve the 3D geometry information of the cup sole shoe type, the lasted upper would be scanned from three different angles. Figure 9 (a) presents the three scanned postures of the cup sole lasted upper, respectively are right, bottom, and left. After the geometric information of these three postures is obtained, the synthesis diagram is shown as Fig. 9 (b) and saved for later trajectory compensation. 


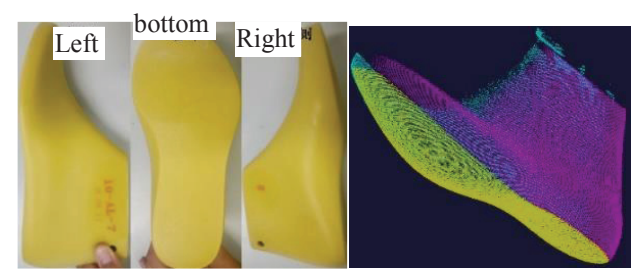

(a)

(b)

Fig. 9. Scan Postures of cup sole shoe type: (a) Three Postures, and (b) 3D Fitted Plot.

\section{Trajectory compensation and modification}

Sport shoes is the one that require the highest functionality among all shoe types; hence, the materials for the upper, except leather or fabric, would design to use foam to rise the comfortability of wearing, or add the supported hard structure, such as stiffener in back parts, to create additional support. Due to the combination of different materials in upper manufacturing, it causes differences even to the same size of the same shoe type.

Therefore, the precious working trajectory of shoes like these cannot be obtained directly and only with the 3D shoe upper model; thus, the assistance of $3 \mathrm{D}$ visualization system is required to retrieve the real geometry information of every shoe. Further, via the algorithm to calculated and then compensate the differences of each shoe upper, and then the robot arm is possible to correctly execute the process of roughing and cementing.

The compensation process is descripted as follows.

1. Read the above stated scanned data of two sides scanning of the lasted upper.

2. Construct the data of the lasted upper to $\mathrm{KD}$ tree format.

3. Search 3 nearest scanned points of each standard contour point in KD tree.

4. Project each standard contour point to the surface constructed by the 3 nearest scanned points, and thus the compensated contact points of the roughing and cementing trajectory are obtained.

5. Fit the compensated points to NURBS curves, smooth the curves, and generate the new contact points.

6. Calculate the origin coordinator of the end effector with Rx, Ry, and $\mathrm{Rz}$ according to the transformation matrix of the robot end effector.

7. Output the JBI file for the robot arm trajectory.

\subsection{KD tree data structure}

KD Tree is a space-partitioning data structure for organizing points in a k-dimensional space. $\mathrm{KD}$ tree structure is characterized to fast search the nearest neighbor in space, which is often applied to search the nearest adjacent point to the specific point group. As shown in Fig. 10, for the 2-dimensional point data, KD Tree would separate the points into two sides. The points greater than the root are in the right side, and each node will be separated in the next level of dimension till all the levels of dimensions used and the classification is completed [9].

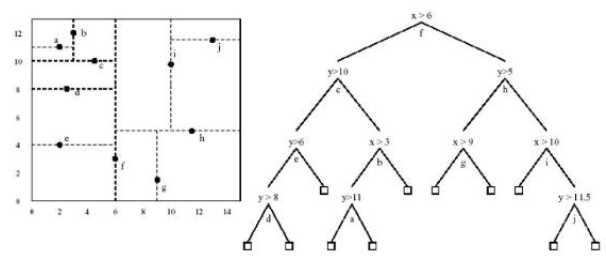

Fig. 10. 2-Dimension KD Tree [10].

\subsection{Contact Point Compensation on 3D Fitted Plot}

Because there are errors existed between the contact point of the 3D scanned data and the existed 3D model, this study compensated the errors by projecting the contact points at its nearest scanned point. Via the above mentioned KD tree format, on the scanned data of the lasted upper, three nearest neighbor points to the original contact point are searched and constructed a surface; and then the new compensated contact point is calculated as shown in Fig. 11. Figure 12 presents the result that all the contact points of the roughing and cementing contour are compensated.

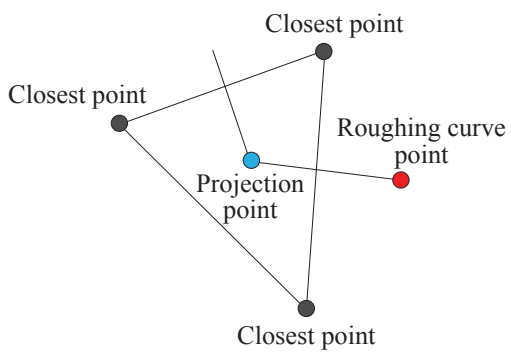

Fig. 11. Diagram of Contact Point Projected to Surface Constructed by Three Neighbor Points.

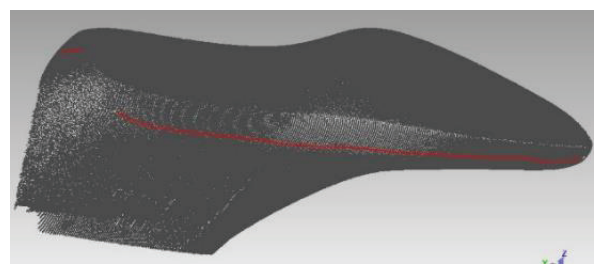

Fig. 12. New contact points after compensation.

\subsection{Fitting and smoothing of NURBS curve}

Figure 13 shows the contact points of the contour line after compensation, which presents to be a fluctuant line. If these contact points are directly converted to the working trajectory of robot arm that might cause vibration to the robot arm because of an uneven route. 
Therefore, before the calculation of trajectory conversion, smoothing is required. In this study, the non-smooth contact points were fitted to Non-uniform Rational B-spline (NURBS) curve, and then the contact points were rearranged in accordance with the fitted curve to generated new contact points after smoothing.

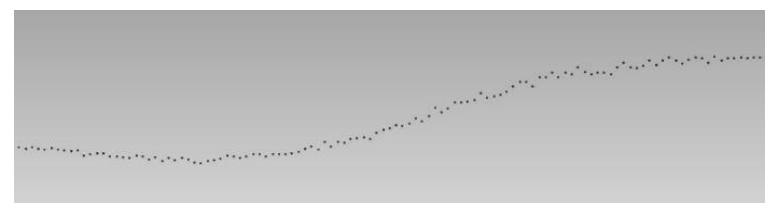

Fig. 13. Enlarged Segment of Compensated contact Points Before Smoothing.

The mathematic definition of Non-uniform Rational B-spline (NURBS) is descripted as Eqs (1) to (4), where $\mathrm{n}+1$ is the number of control factor, $k$ number of the contact points, $\mathrm{N}_{\mathrm{i}, \mathrm{k}}$ the basic function of $\mathrm{B}$-spline, $\mathrm{P}_{\mathrm{i}}$ control points, and $\mathrm{u}$ the parameter value.

$C(u)=\sum_{i=0}^{n} N_{i, k} P_{i}$

$N_{i, 0}(u)=\left\{\begin{array}{l}1, \text { if } \mathrm{u}_{\mathrm{i}} \leq u<u_{i+1} \\ 0, \text { otherwise }\end{array}\right.$

$N_{i, p}(u)=\frac{u-u_{i}}{u_{i+p}-u_{i}} N_{i, p-1}(u)+\frac{u_{i+p+1}-u}{u_{i+p+1}-u_{i+1}} N_{i+1, p-1}(u)$

The curve fitting is processed by matching to a $C\left(u_{j}\right)$ NURBS curve to make the contact point $q_{j}$ and the curve to have the smallest error $e$ calculated as Eq. (4).

$e=\sum_{j=0}^{m}\left[c\left(\bar{u}_{j}\right)-q_{j}\right]^{2}=\sum_{j=0}^{n}\left[\sum_{i=0}^{n} N_{i, k} p_{i}-q_{j}\right]^{2}$

That is, if $n$ and $k$ are set to be constant, the control point of $P_{i}$ curve is obtained by calculating the extremum of $\min (e)=0$. And then the fitted curve is used to smooth $u$ and re-distribute the contact points, thus the new smoothed contact points are acquired shown as Fig. 14.

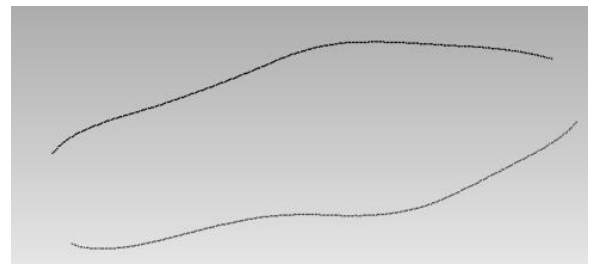

Fig. 14. Smoothed Contact Points for Roughing and Cementing.

\section{Robot arm}

The robot arm employed in this study is Yaskawa MH6, which is an 6-axis articulated manipulator, portable wight $6 \mathrm{~kg}$, and repeatbility $\pm 0.08 \mathrm{~mm}$. The research system setup is shown as Fig. 2.

\section{Experimental result and discussion}

In this study, the path is designed by the plug-in program based on Rhino CAD platform, and its data is generated automatically as shown in Figure 15. Each contact point data contain position components $(\mathrm{x}, \mathrm{y}, \mathrm{z})$ and orientation components ( $\mathrm{Rx}, \mathrm{Ry}, \mathrm{Rz}$ ) arranged in a row. The six values represent $\mathrm{x}, \mathrm{y}, \mathrm{z}, \mathrm{Rx}, \mathrm{Ry}$ and $\mathrm{Rz}$ from left to right. In addition, the units of position and orientation are in [mm] and [degree] respectively.

\begin{tabular}{|cccccccc|}
$\mathrm{X}$ & $\mathrm{Y}$ & $\mathrm{Z}$ & $\mathrm{Rx}$ & $\mathrm{Ry}$ & $\mathrm{Rz}$ \\
\hline
\end{tabular}

Fig. 15. Format of Contact Points.

Four Snapshots in Figure 16 show the proposed system implementing the processes of roughing and cementing for a cup sole shoe type after the integration of hardware and software. The white blush is used as roughing tool. According to a path, the blush can contact the shoe well along the curve during 1st snapshot $\rightarrow$ 2nd snapshot and 3rd snapshot $\rightarrow$ th snapshot. However, the shoe runs an unexpected path and pushes the blush suddenly during 2 nd and 3 rd snapshots, when the robot changes its orientation to achieve the desired contact angle. Thus, it should be interpolated some transition points to escape collisions.

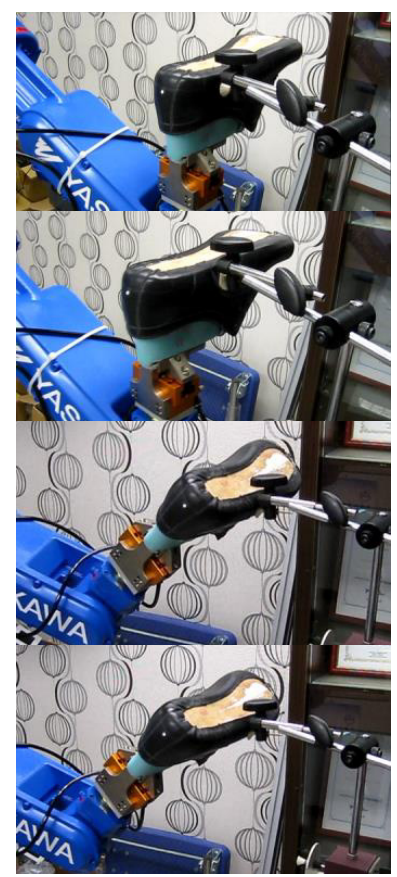

Fig. 16. Testing of Proposed System Implementing Roughing and Cementing on Cup Sole Shoe Type.

In the plug-in program, it is assumed that the shoe contact with a roughing tool at a sharp point, and the 
contact force is omitted. In the experiment, the contact is a plane, and it has an obvious friction force. Therefore, the blush is damaged over time.

\section{Conclusion}

Designated to the current shoe industry situation of customization, small quantity, and variation of shoe types, in this study, the automatic robot arm is developed, which is capable to automatically compensate errors between 3D model and each lasted upper and able to implement the processes of roughing and cementing on the cup sole shoe type. The developed plug-in program for Rhino 3D CAD platform enables the contour planning for roughing and cementing on $3 \mathrm{D}$ graphic interface, the calculation of the compensated contact points with the real-time $3 \mathrm{D}$ scanned information, and generation of working trajectory of Yaskawa robot arm after smoothing and coordinator conversion. Thus the employed robot arm are successfully driven to implements the roughing and cementing. The proposed system is potential to further development, such as equipping force sensors on the robot arm to acquire the value of the force applied on roughing shoe uppers for advanced adjustment of the roughing position for the purpose of higher accuracy on error compensation.

\section{References}

1. http://www.adidas.com/us/originals-shoes

2. http://www.nike-taiwan.com.tw/

3. Chuanyu Wu, Yong Ni, Qinchuan Li, Yuzhen Jin, Implementation of 5-DOF apparatus used for adhesive spray and roughing of shoe upper, Proceedings of the IEEE International Conference on Automation and Logistics, Qingdao, China, pp. 1408-1413, September 200.

4. Yuzhen Jin, Lei Zhang, Chuanyu Wu, Zuchao Zhu, Detection of 3D curve for shoe sole spraying based on laser triangulation measurement, Proceedings of the IEEE International Conference on Automation and Logistics, Shenyang, China, pp. 865-868, 2009

5. Chuanyu Wu, Xiaojie Mao, Xiangjun Shi, Lei Zhang, Methods of generating robot spraying trajectory based on shoe sole information, Intelligent systems and applications, ISA 2009. International Workshop, pp. 1-4, 2009

6. Francesco Jatta, Lorenzo Zanoni, Irene Fassi, S. Negri, A roughing/cementing robotic cell for custom made shoe manufacture, Int. J. Computer Integrated Manufacturing, 17(7), pp. 645-652, 2004

7. Z. Hu, C. Marshall, R. Bicker, P. Taylor, Automatic surface roughing with 3D machine vision and cooperative robot control, Robotics and Autonomous Systems, 55(7), pp. 552-560, 2007

8. W. T. Wang, T. Y. Lee, C. M. Yang, W. S. Yin, Research of 3D vision intelligent robot system for shoe roughing and cementing, Proceedings of 2015 CSME, 2015/12

9. T. H. Chu, H. Kuo, W. Su, Cloud point PCL Learning, Beijing University of Aeronautics and Astronautics Press, 2012/10

10. https://en.wikipedia.org/wiki/K-d tree 